\title{
Particle formation and risk of embolization during transseptal catheterization: comparison of standard transseptal needles and a new radiofrequency transseptal needle
}

\author{
Gregory K. Feld • Jay Tiongson • Ganiyu Oshodi
}

Received: 29 April 2010 / Accepted: 19 November 2010/Published online: 20 January 2011

(C) The Author(s) 2011. This article is published with open access at Springerlink.com

\begin{abstract}
Objective Anecdotally, the Brockenbrough transseptal needle generates plastic particles through a process of skiving (shaving off particles), when advanced through the dilator and sheath. This study was performed to assess particle creation by the Brockenbrough needle during transseptal catheterization. We explore strategies that may reduce this phenomenon, including use of the Brockenbrough stylet and a radiofrequency transseptal needle.

Method In vitro simulations of transseptal catheterization were performed using Brockenbrough transseptal needles and a new radiofrequency transseptal needle. Particles that were created during advancement of transseptal needles through the sheath and dilator were collected and analyzed. Particles in the visible range of $50 \mu \mathrm{m}$ to $4 \mathrm{~mm}$ were identified using a light microscope, whereas particles in the sub-visible, yet clinically relevant range of 10 to $50 \mu \mathrm{m}$, were counted using a light obscuration method.

Results All simulated procedures using the Brockenbrough transseptal needles, with or without a stylet, generated visible particles. Simulated procedures with the radiofrequency transseptal needle generated no visible particles. A greater number of sub-visible particles were generated with the standard Brockenbrough transseptal needle (BKR-1) without stylet compared with the standard Brockenbrough needle (BRK-1) with stylet, the Brockenbrough extra sharp (BRK-1XS) needle with or without stylet, and the radiofrequency needle (NRG C1).
\end{abstract}

\footnotetext{
G. K. Feld $(\bowtie) \cdot$ J. Tiongson $\cdot$ G. Oshodi

Department of Medicine, Division of Cardiology,

Cardiac Electrophysiology Program UCSD Medical Center,

4169 Front St.,

San Diego, CA 92103-8649, USA

e-mail: gfeld@ucsd.edu
}

Conclusion Clinically relevant particles, both visible and sub-visible, with the potential for causing embolic complications, are generated by the BRK-1 needle without stylet. Use of a stylet in the BRK-1 needle, or the BRK-1XS needle with or without stylet, appears to reduce the size and amount of particles created. The NRG $\mathrm{C} 1$ needle appears to eliminate visible particles and is comparable to the BRK-1 with stylet and the BRK-1XS with or without stylet in generation of subvisible particles. Important steps can be taken to minimize the creation of particles during the advancement of the BRK-1 through the transseptal sheath and dilator.

Keywords Transseptal puncture · Skiving · Radiofrequency (RF) needle $\cdot$ Brockenbrough needle

\section{Background}

$\mathrm{T}$ transseptal catheterization is the technique of choice for left atrial access for the treatment of several conditions. Ross et al. pioneered the transseptal technique in the $1960 \mathrm{~s}$ [1]. Brockenbrough described a refined technique in 1962 [2]. Since then, there has been little variation in the technique [3].

Earley et al. describe the transseptal procedure in greater detail [4]. Central to the transseptal procedure is a sharptipped needle, commonly referred to as the Brockenbrough needle, which is used in combination with a sheath and dilator. There are two common techniques used to load the Brockenbrough needle into the dilator, one with the stylet in place and one with the stylet removed. Elimination of the stylet with connection of the needle to a pressure transducer directly before advancing it through the dilator may speed up the procedure. However, this exposes the sharp tip of the needle as it is advanced through the dilator. 
Recently, the number of transseptal catheterization procedures performed annually has increased as a result of an increase in left-sided procedures such as atrial fibrillation ablation. Despite the relative safety of this procedure, there are still potential complications associated with the technique, which include cardiac tamponade, air embolism, and thromboembolism due to clot formation or tissue charring during ablation [5]. Thromboembolism due to a previously unreported cause may also result from the creation of plastic particles that are scraped off the inner wall of the transseptal dilator by the Brockenbrough transseptal needle as it is advanced through the dilator.

Awareness of the risk of creating particles first emerged at our center during a routine transseptal catheterization procedure. After left atrial access had been successfully achieved, contrast media could not be injected through the Brockenbrough needle. We therefore removed the needle, and while maintaining the position of the tip of the dilator inside the left atrium, we attempted to advance a guidewire through the dilator into the left atrium. However, the guidewire could not be advanced through the dilator either. This required that the dilator be removed from the sheath, and when the dilator was flushed, a large piece of shaved plastic was ejected from the lumen, which it had been obstructing.

Particles introduced into the circulatory system may pose a risk to patient health. Particles as small as $15-50 \mu \mathrm{m}$ can obstruct the microvascular bed causing microinfarcts and left ventricular dysfunction [6]. A known standard for assessing the risk from particles is the United States Pharmacopeia USP 788 "Particulate matter in injections," which illustrates that particles that are greater than $10 \mu \mathrm{m}$ in size pose a clinically significant risk to patient health, and particles that are greater than $25 \mu \mathrm{m}$ pose a high risk to the patient. [6-8]

The objectives of this study were to measure the creation of skived particles through in vitro testing of two common Brockenbrough transseptal needles, to evaluate if certain techniques or device features had an impact on the creation of particles, and to evaluate a new radiofrequency transseptal needle [9], to determine if its rounded distal tip had an impact on the creation of particles.

\section{Methods}

\subsection{Device selection}

Three transseptal needles were tested as shown in Fig. 1. The needles all had similar distal curves so as to allow for a direct comparison of the test results. One model of sheath and dilator was used for the study.
The tests for visible particles were conducted with the BRK-1 and BRK-1XS Brockenbrough needles (St. Jude, Inc., Minneapolis, MN) and the NRG C1 RF needle (Baylis Medical, Inc., Montreal, QC, Canada). Both the BRK-1 and the BRK-1XS were tested with and without a stylet. The BRK-1 needle has a bevel that is in-line with the curve of needle whereas the BRK-1XS has a bevel that is opposite to the curve of the needle. Both needles were tested to determine if the bevel orientation has an impact on particle generation. The RG C1 RF needle has a closed rounded tip with two side holes located $2 \mathrm{~mm}$ from the distal tip through which pressure is measured and contrast is injected, and therefore, does not accept a stylet. All tests were conducted using a new \#8-French Daig SL-1 transseptal sheath and dilator (St. Jude, Inc., Minneapolis, MN).

\subsection{Transseptal procedure simulation}

All devices tested were sterile, and previously unused. While it is assumed that sterile products come completely particlefree out of the package, this is not always the case as there may be sub-visible particles present. For this reason, particle-free saline was flushed through each sterile sheath kit, and over and through each needle to clean away preexisting sub-visible particles. The flushed fluid was captured and measured to establish a baseline sub-visible particle measurement for each set of devices. There were no visible particles present on any devices tested prior to their use.

The introducer sheath was inserted into $3 / 8$ " clear vinyl tubing to mimic the constraints that the vasculature imposes

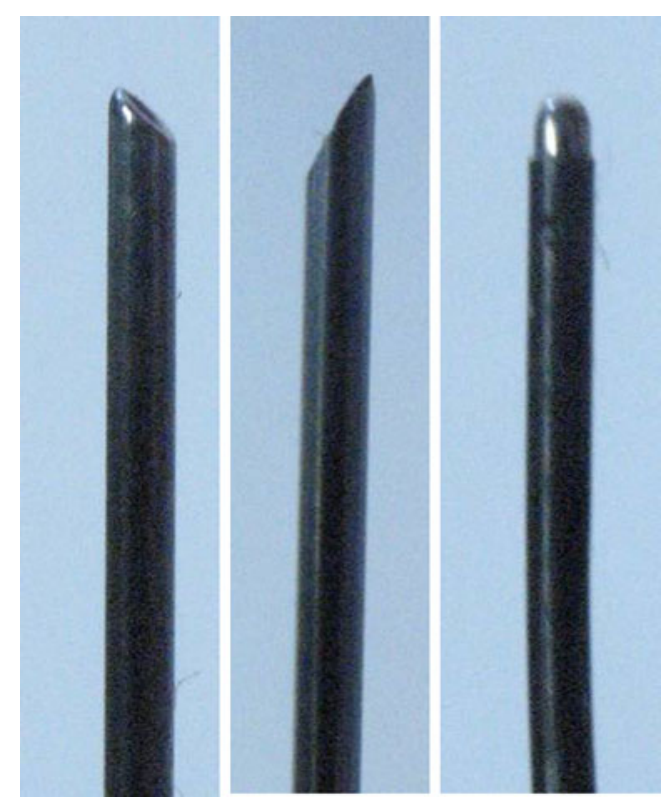

Fig. 1 Transseptal needles evaluated from left to right are the BRK-1, BRK-1XS, NRG-C1 
upon the introducer kit. Two sets of tests were conducted, one to determine the creation of visible particles which are greater than $50 \mathrm{um}$, and one to determine the creation of clinically significant sub-visible particles which are in the range of 10 to $50 \mathrm{um}$.

Tests for the visible particles consisted of advancing the needle back and forth three times through the sheath and dilator followed by flushing the assembly. The resulting solution was filtered through millipore filter paper $(47 \mathrm{~mm}$, $0.8 \mathrm{um}$, black grid, Type AA) and allowed to dry in air. Each filter was examined using a reflected light compound microscope following the microscopic particle count test as described in the United States Pharmacopeia USP 788 guidance document. Visible particles were photographed using a Zeiss Stereo Microscope.

The tests for the sub-visible particles $(10 \mu \mathrm{m}$ to $50 \mu \mathrm{m})$ used a similar approach. The needle was advanced back and forth three times through the sheath and dilator, after which the assembly was flushed and the resulting solution was captured for measurement. In order to reduce the chance of particulate leftover during packaging from affecting the results of the study comparison, the dilators and sheaths were thoroughly flushed with sterile saline $\left(8-10 \mathrm{~cm}^{3}\right)$ prior to testing. Each solution suspension was degassed for $2 \mathrm{~min}$ prior to analysis. Measurements of the sub-visible particles were conducted using the light obscuration method, by means of a HIAC Royco Particle Counter. The light obscuration method consists of passing the solution through a laser beam. Particles obscure the laser beam during its transit through the solution and the total laser light minus the light obscured by the particles is used by a particle counter to count and size the particles. Each sample was measured four times using a sample volume of $5 \mathrm{~mL}$. The data from the first measurement was discarded and the average of the last three samples is reported. This method is described in the United States Pharmacopeia USP 788 guidance document.

\subsection{Statistical analysis}

Statistical analysis of the difference in means in the subvisible particle counts (Table 1) was performed using a one-way ANOVA with Bonferroni correction for multiple comparisons with SPSS statistical software package (SPSS, Inc., Chicago, IL).

\section{Results}

Skived particles were created by the tip of the Brockenbrough needle scraping against the inner wall of the dilator as it was advanced. Scraping was felt through the Brockenbrough needle as an increase in resistance or a grinding sensation during needle advancement. This resistance was particularly noticeable at two sites, (1) when the needle is advanced through the proximal solid hub of the sheath and dilator and (2) when the needle navigates the distal curve of the sheath and dilator. The solid hub of the sheath and dilator imposes a narrow, noncompliant channel in which the curved needle is forcibly straightened. The preset curve at the distal end of the sheath and dilator tip also presents a place where the needle not only actively scrapes the inner wall, but also where in extreme cases it may puncture the sheath and dilator.

There were four separate tests conducted with the Brockenbrough needle, (1) BRK-1 without stylet, (2) BRK-1 with stylet, (3) BRK-1XS without stylet, and (4) BRK-1XS with stylet, all of which generated visible particles. The procedure performed with the NRG C1 radiofrequency needle did not generate any visible particles. Visible particles were found on the outer surface of the Brockenbrough needles, occluding their lumens, and in saline flushed through the dilators after the needles were removed. Particles found on the outer surfaces or flushed from the dilators appeared like twisted lengths of ribbon (Fig. 2). Particles occluding the lumen had the appearance of cylindrical plugs, similar to what might be generated by a biopsy needle (Fig. 3). Interestingly, Wong et al. reported using a transseptal needle specifically for the purpose of liver biopsy [10]. Figure 4 illustrates a variety of particles formed by the Brockenbrough needles during the procedure. Particles measured as large as $6 \mathrm{~mm}$ in length (when untwisted), and over $0.3 \mathrm{~mm}$ in width. The BRK-1 needle without stylet produced the largest size particles. Figure 4 also illustrates that there were no visible particles created by the RF needle.

The BRK-1 needle without a stylet generated a significantly greater number of sub-visible particles (Table 1) compared with the BRK-1 needle with stylet, the BRK-1XS needle with or without stylet, or the NRG C1 radiofrequency needle $(p<0.01$ for BRK-1 without stylet vs. all others).

Table 1 Light obscuration results for sub-visible particles $10-50 \mu \mathrm{m}$

\begin{tabular}{|c|c|c|c|c|c|}
\hline & BRK-1 without stylet & BRK-1 with stylet & BRK-1XS without stylet & BRK-1XS with stylet & NRG C1 \\
\hline Post-procedure particle count & $50.4 \pm 1.49^{*}$ & $12.9 \pm 2.25$ & $10.8 \pm 0.67$ & $15.6 \pm 1.1$ & $17.2 \pm 2.9$ \\
\hline
\end{tabular}




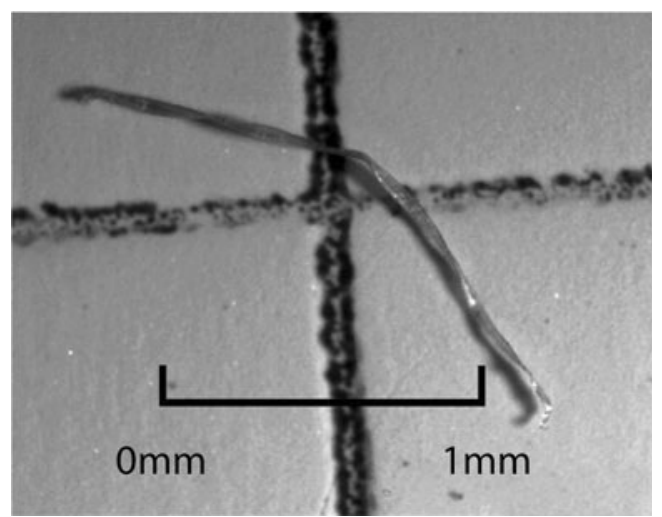

Fig. 2 Shaved particle in the visible range

\section{Discussion}

Unlike other complications associated with transseptal catheterization procedures, the introduction of particles can be asymptomatic at first, particularly if the patient is sedated or under general anesthesia. When signs or symptoms of a systemic embolic event are finally recognized, they are typically attributed to thromboembolism, rather than particle embolization $[11,12]$. Clinical experience and the bench-top testing in this report confirm the generation of particles that could potentially cause an embolism during transseptal catheterization procedures. Systemic embolism has long been considered a Major Adverse Cardiac Event during angioplasty [6-8].

As with any needle designed to puncture the atrial septum with mechanical force, sharpness is critical for the needle to be effective. There is a direct trade-off however between the sharpness of the needle required to make the puncture and the degree to which it scrapes off particles from the inner wall of the dilator through which it is advanced to perform the puncture. New developments in needle tip design have recently become available, focusing on bevel orientation (BRK-1XS) and rounded atraumatic tips (NRG C1). In this study, the BRK-1XS had mixed results, with no impact on the creation of visible particles, while reducing the amount of sub-visible particles compared with the standard BRK-1 needle. The NRG C1 needle, which uses radiofrequency energy to create the transseptal puncture, appears to eliminate the creation of visible particles and is comparable with the BRX-1XS needle with or without a stylet and BRX-1 needle with stylet in creating very few sub-visible particles.

In addition to specific needle selection, several other strategies can be used to minimize the creation of particles during transseptal needle advancement through the sheath and dilator. First, it is important that the needle be allowed to rotate freely within the dilator as it is advanced through the tortuous route imposed by the venous system, particularly in the pelvic area. Failure to allow for this rotation, which may be up to $360^{\circ}$ in some cases, increases the chance that the tip of the Brockenbrough needle will scrape against the inner lumen of the dilator, thereby increasing the possibility of skiving of plastic particles or, in extreme cases, puncturing through the dilator and sheath wall. The use of a stylet with the BRK-1 needle did not eliminate visible particles, but did reduce the amount of the subvisible particles created when compared with the tests done without the stylet. However, this approach results in the need to aspirate the needle to eliminate air once deployed in the sheath, which is difficult in some cases because of a lack of airtight seal between the needle and dilator. It is also common practice in some centers to flush the transseptal needle during or after its advancement into the dilator, but prior to transseptal puncture. Flushing the needle in the right atrium may expel particles prior to crossing into the left atrium where they pose a potentially greater risk, but it is not known what adverse effect this may have on the pulmonary circulation long term. It may also be beneficial to flush the needle as it is advanced past the hub and the curve in the sheath and dilator. Another technique that some practitioners employ, in an effort to loosen and expel particles that may be susceptible to skiving, is to repeatedly insert and remove the needle from the dilator in order to scrape out and clear the inner lumen, while the needle/dilator assembly is outside the body. The needle/dilator assembly, attached to a pressure transducer, is then inserted into the transseptal sheath, which is already positioned in the right atrium prior to transseptal puncture.

Practitioners frequently need to re-shape the curve of the available transseptal needles depending on the anatomy of the patient so as to better engage the inter-atrial septum. Currently, needles with larger curves are available, which eliminate the need for reshaping. But in either case, if the needle is reshaped with a larger curve or a large curve needle is required to perform the transseptal puncture, this will increase the forces applied by the tip of the needle as it is advanced through the dilator against the inner lumen, which in turn may increase the risk of skiving particles.

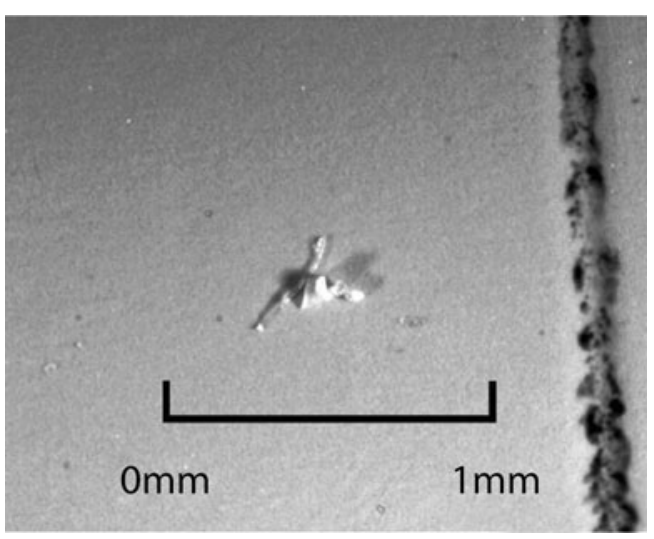

Fig. 3 Cored particle in the visible range 
Fig. 4 Various visible particles observed following three insertions with each needle type

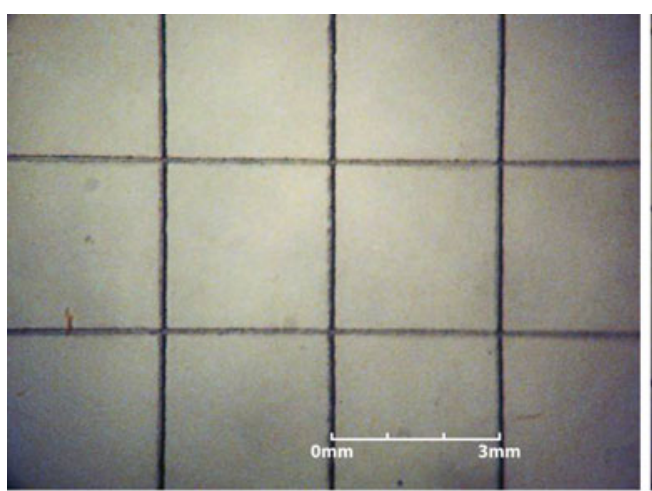

NRG C1

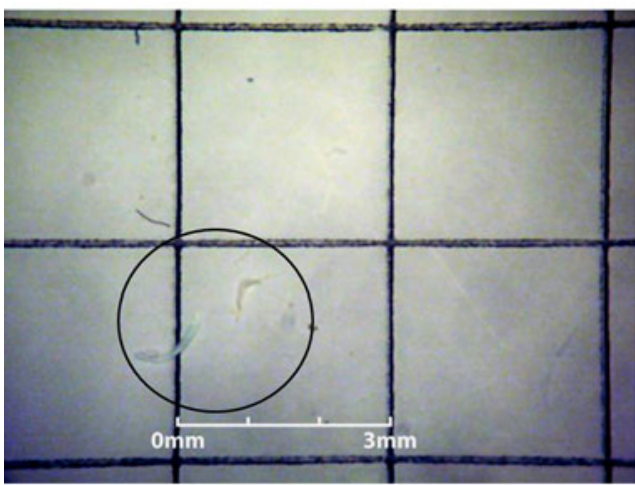

BRK-1 with stylet

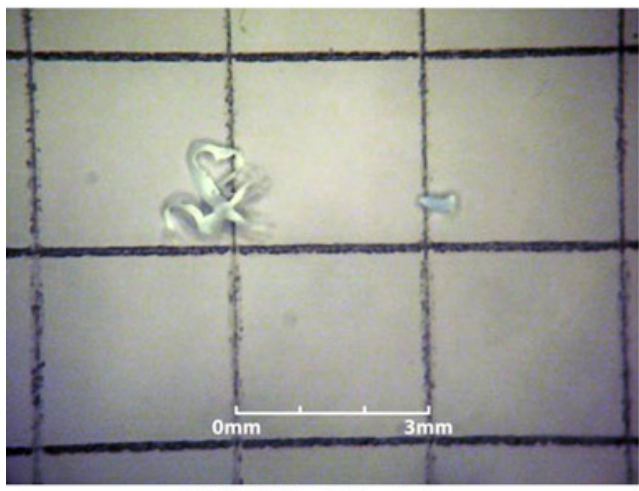

BRK-1XS with stylet

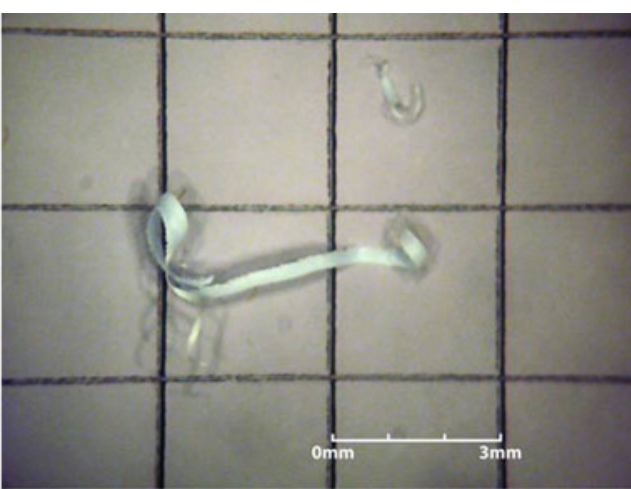

BRK-1

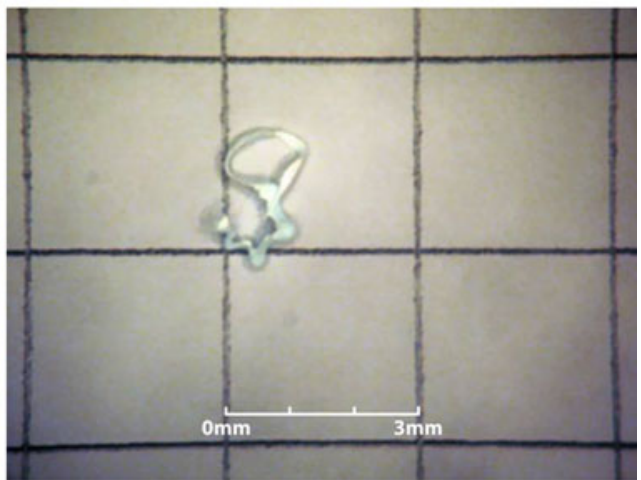

BRK-1XS

\section{Study limitations}

It was beyond the scope of this study to examine all potential combinations of Brockenbrough needles, transseptal sheaths, and dilators. The BRK-1 and BRK-1XS needles were chosen for testing along with the Daig SL-1 introducer kit because of their widespread use. The NRG $\mathrm{C} 1$ radiofrequency needle was chosen for testing as it offers a rounded atraumatic tip. It is acknowledged that there are a number of other suppliers of Brockenbrough needles, sheaths, and dilators. Different needles may be more or less sharp, may have a more or less acute curve, and may have a stiffer or softer shaft than those tested, which in turn could result in variations on the creation of particles. As well, the size and suppleness of the sheath and dilator can also be expected to have an impact. A more compliant sheath and dilator may result in less particle creation, whereas a stiffer sheath and dilator may result in greater particle creation. Therefore, the results and conclusions may not be extrapolated to all devices currently available. In a clinical setting, the sheath and dilator are physically constrained by the vasculature that changes with respect to each patient's anatomy. Thus, it is possible that during clinical use a sheath and dilator may be more or less compliant and perhaps more or less prone to skiving than observed during the experimental conditions in this study. A small sample size of devices was used in this study, which may have limited to some extent the statistical analysis of the data. 


\section{Conclusions}

The Brockenbrough needle creates clinically significant visible particles when advanced through a transseptal sheath and dilator. This is true whether the standard stylet is used or not. However, the use of a stylet with the BRK-1 needle or a BRK-1XS needle with or without stylet was effective in reducing the number of clinically significant sub-visible particles. The NRG C1 radiofrequency needle, with a rounded atraumatic tip, does not create any clinically significant visible particles and very few sub-visible particles when advanced through a transseptal sheath and dilator.

As the dangers of embolization during transseptal catheterization are well-known, additional steps may need to be taken to mitigate the risk of particle creation. It is important that the needle be allowed to rotate within the dilator as it is advanced as this alleviates the pressure of the needle against the inner wall of the dilator. Reaming the dilator with the needle outside the body and loading the needle/dilator assembly as a single unit into the sheath may also reduce the amount of particles introduced into the body. Further evaluation of the various needle, sheath, and dilator combinations used at any specific institution for potential skiving of particles could be done prior to transseptal catheterization as well.

Acknowledgments This study was supported in part by Baylis Medical, Inc., Vancouver, British Columbia, Canada.

Open Access This article is distributed under the terms of the Creative Commons Attribution Noncommercial License which permits any noncommercial use, distribution, and reproduction in any medium, provided the original author(s) and source are credited.

\section{References}

1. Ross, J., Jr., Braunwald, E., \& Morrow, A. (1960). Left heart catheterization by the transseptal route: a description of the technic and its applications. Circulation, 22, 927-934.

2. Brockenbrough, E. C., Braunwald, E., \& Ross, J., Jr. (1962). Transseptal left heart catheterization. A review of 450 studies and description of an improved technic. Circulation, 25, 15-21.

3. Ross, J., Jr. (2008). Transseptal left heart catheterization a 50-year odyssey. Journal of the American College of Cardiology, 51, $2107-2115$

4. Earley, M. J. (2009). How to perform a transseptal puncture. Heart, 95, 85-92.

5. Gaita, F., Caponi, D., Pianelli, M., Scaglione, M., Toso, E., Cesarani, F., et al. (2010). Radiofrequency catheter ablation of atrial fibrillation: a cause of silent thromboembolism? Magnetic resonance imaging assessment of cerebral thromboembolism in patients undergoing ablation of atrial fibrillation. Circulation, 122, 1667-1673.

6. Sangiorgi, G., \& Colombo, A. (2003). Distal protection devices. Heart Sep, 89, 990-992.

7. Biam, D. S., et al. (2002). Randomized trial of a distal embolic protection device during percutaneous intervention of saphenous vein aorto-coronary bypass grafts. Circulation, 105, 1285.

8. Stone, G. W., Rogers, C., Hermiller, J., Feldman, R., Hall, P., Haber, R., et al. (2003). Randomized comparison of distal protection with a filter-based catheter and a balloon occlusion and aspiration system during percutaneous intervention of diseased saphenous vein aortocoronary bypass grafts. Circulation, 108, 548-553.

9. Smelley, M. P., Shah, D. P., Weisberg, I., Kim, S. S., Lin, A. C., Beshai, J. F., et al. (2010). Initial experience using a radiofrequency powered transseptal needle. Journal of Cardiovascular Electrophysiology, 21, 423-427.

10. Wong, C. W., Ruygrok, P., Sutton, T., Ding, P., van Vliet, C., Occleshaw, C., et al. (2010). Transseptal fine needle aspiration of a large left atrial tumour. Heart, Lung \& Circulation, 19, 438-439.

11. Maleki, K., Mohammadi, R., Hart, D., Cotiga, D., Farhat, N., \& Steinberg, J. S. (2005). Intracardiac ultrasound detection of thrombus on transseptal sheath: incidence, treatment, and prevention. Journal of Cardiovascular Electrophysiology, 16, 561-565.

12. Romero-Rodriguez, N., Pedrote, A., Arana-Rueda, E., \& MogollonJimenez, M. V. (2008). Thrombus development on a transseptal sheath in the right atrium before electrical pulmonary vein isolation. Indian Pacing and Electrophysiology Journal, 8, 72-74. 